Ann. Génét. Sél. anim., r969, 1 (4), 379-382.

\title{
LA STÉRILITÉ DES MÂLES DANS LES RACES GAPRINES SUISSES SANS CORNES
}

\author{
W. WEBER \\ Institut de Zootechnie, \\ École vétérinaire, Bremgartenstr. тоgа, зог а Berne, Suisse
}

\section{RÉSUMÉ}

966 jeunes boucs de trois races suisses sans cornes (Gessenay, Oberhasli-Brienz et Toggenbourg) ont été examinés lors des concours centraux annuels de ces races, de 1957 à $1968: 3,4$ p. 100 d'entre eux présentaient une hypoplasie des testicules, 13,4 p. 100 une obstruction uni- ou bilatérale de l'épididyme. Les boucs hypoplasiques ainsi que les sténosiques bilatéraux sont stériles. Comme un certain nombre d'individus anormaux sont détectés précocement par les éleveurs et éliminés, les chiffres présentés, qui varient d'ailleurs d'une race à l'autre, doivent être considérés comme des estimations par défaut des fréquences réelles. 175 boucs témoins observés dans la race cornue Verzasca étaient indemnes de toute anomalie génitale.

\section{INTRODUCTION}

La Suisse possède 4 races de Chèvres sans cornes : Gessenay (=Saanen), Toggenbourg, Oberhasli-Brienz et Appenzell. Pour les 3 premières, il existe tous les ans des concours où sont présentés les reproducteurs âgés de 5 mois à 4 ans. Ces concours ont lieu au mois de septembre à Thoune pour la race Saanen, à Sargans pour le Toggenbourg et à Interlaken pour l'Oberhasli. A l'occasion de ces concours qui sont en réalité des marchés-concours les animaux correspondant au but d'élevage sont inscrits au herdbook. Depuis I957, à la suite des travaux de LöLIGER sur la stérilité des boucs, travaux en partie réalisés en Suisse (1953, I956a et $b$, I957 $a$ et $b$ ), on pratique un certain nombre d'examens systématiques sur tous les mâles de ces concours afin de détecter les animaux stériles.

\section{I. -- LES EXAMENS}

Deux anomalies sont détectables : 1'hypoplasie des testicules et la congestion séminale.

L'hypoplasie des testicules se manifeste très tôt après la descente des testicules, qui sont de consistance molle et de la taille d'une noix ou d'une noisette. 
Le scrotum est également réduit et les animaux ne présentent aucun instinct sexuel. Il n'y a jamais de production de semence. L'anomalie est donc congénitale.

Les symptômes de la congestion séminale qui, généralement, n'apparaissent pas à la naissance sont les suivants : dans la région de la tête de l'épididyme, plus rarement dans le corps ou la queue, on trouve des nodules durs, indolores ayant jusqu'à la taille d'une noix. Le testicule est de taille et de consistance normales. L'instinct sexuel est également normal. Les nodules dans la région de la tête de l'épididyme obstruent mécaniquement le passage du sperme, il en résulte une stérilité secondaire si, comme cela se produit parfois, l'anomalie est bilatérale. Lorsqu'elle est unilatérale, l'animal est fertile et peut le rester toute sa vie. Parfois, cependant la congestion séminale se développe de l'autre côté l'année suivante. Il semble que la congestion séminale fasse son apparition principalement à la puberté ou, parfois, un peu après. A l'aide d'un vagin artificiel on collecte le sperme des animaux atteints uni- ou bilatéralement. L'éjaculat des boucs encore fertiles est jaunâtre et opaque, celui des boucs stériles aqueux et transparent, sans spermatozoïdes.

\section{II. - LES RÉSULTATS}

Des résultats partiels, portant sur les jeunes mâles pour les années I957-6I et 62-67, ont été déjà présentés (WEBER, I96I, I967); on les a rassemblés dans le tableau I, leur adjoignant les résultats de 1968 .

Comme témoins cornus, I 75 mâles de la race Verzasca noire cornue ont été examinés ces dernières années lors de ces mêmes concours. Tous étaient parfaitement normaux.

\section{III. - DISCUSSION}

La fréquence générale de 3,4 p. Ioo pour l'hypoplasie dépend surtout des boucs de Toggenbourg, assez peu de ceux d'Oberhasli-Brienz chez lesquels, pourtant, la fréquence serait peut-être plus haute mais les éleveurs savent remarquer, semble-t-i1, le sous-développement des organes génitaux et n'exposent pas les sujets qui le présentent.

La fréquence générale des boucs refusés pour congestion séminale uni- ou bilatérale est de 13,4 p. Ioo. Ce chiffre est certainement inférieur à la réalité car, comme pour 1'hypoplasie, au cours des ans certains éleveurs ont appris à détecter de tels animaux et ne les exposent pas. On remarque les plus hautes fréquences chez la race Oberhasli-Brienz mais, vu le faible effectif total observé dans cette race $\left({ }_{156}\right)$ et les remarques formulées ci-dessus, il serait prématuré de conclure que la congestion séminale apparaît plus fréquemment dans cette race que dans les autres.

D'une manière générale donc, les fréquences constatées doivent être considérées comme des estimations par défaut des fréquences réelles. LöLIGER (déjà cité) a été le premier en Suisse à s'intéresser à l'étiologie de la congestion séminale; selon lui, une infection à colibacilles serait très probablement à l'origine de cette malfor- 


\section{TABLEAU I}

Fréquence des animaux atteints d'anomalies testiculaires dans trois races suisses sans cornes

\begin{tabular}{|c|c|c|c|c|}
\hline & \multirow{2}{*}{$\left|\begin{array}{ll}\multicolumn{2}{c}{\text { Total }} \\
\text { des } & \text { jeunes boucs } \\
\text { présentés } \\
\text { (de } 5 \text { à } 12 \text { mois) }\end{array}\right|$} & \multirow{2}{*}{$\begin{array}{l}\text { Hypoplasie } \\
\text { des testicules }\end{array}$} & \multicolumn{2}{|c|}{ Obstruction de l'épididyme } \\
\hline & & & Unilatérale & Bilatérale \\
\hline \multicolumn{5}{|l|}{ Gessenay } \\
\hline I958-6I & I57 & 8 & 7 & $6(3,8 \%)$ \\
\hline $1962-67$ & 204 & 5 & I3 & I5 $(7,3 \%)$ \\
\hline 1968 & 24 & - & I & $I(4,2 \%)$ \\
\hline Total . . . . & 385 & I3 $(3,3 \%)$ & $2 \mathrm{I} \quad(5,5 \%)$ & $22(5,7 \%)$ \\
\hline \multicolumn{5}{|l|}{ Toggenbourg } \\
\hline I957-6I & I8I & 4 & 7 & I $2(6,5 \%)$ \\
\hline $1962-67 . . .$. & 210 & 13 & $\mathrm{r}_{3}$ & $15(7,1 \%)$ \\
\hline . . . . & 34 & 2 & 3 & 4 \\
\hline Total $\cdot \cdot \cdot \cdot \cdot$ & 425 & I9 $(4,5 \%)$ & $23(5,4 \%)$ & $31 \quad(7,3 \%)$ \\
\hline \multicolumn{5}{|l|}{ Oberhasli-Brienz } \\
\hline I957-6I . . . & 75 & 一 & 4 & Io $(13,2 \%)$ \\
\hline $1962-67 . . .$. & 68 & & 7 & $8(\mathrm{I}, 7 \%)$ \\
\hline 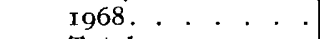 & 13 & - & I & 3 \\
\hline Total . . . . . & I56 & I $(0,7 \%)$ & I2 $(7,7 \%)$ & $2 \mathrm{I}(\mathrm{x} 3,5 \%)$ \\
\hline Totaux. . . . . & 966 & $33(3,4 \%)$ & $56(5,8 \%)$ & $74(7,6 \%)$ \\
\hline
\end{tabular}

mation mais jamais nous n'avons pu obtenir une preuve de la présence d'un germe infectieux dans les organes atteints. Finalement, nos résultats confirment le comportement du gène $P$ déterminant l'absence des cornes, dans 2 de ses effets, tels qu'ils ont été mis en évidence par les travaux allemands et israéliens qu'a résumés LAUVERGNE (I969).

Avec les réserves faites ci-dessus quant à l'obtention des données, il est possible de donner une estimation des 2 paramètres, $\beta_{1}$ et $\beta_{2}$, définis par le dernier auteur cité, qui sont les expressivités des atteintes partielles (la fertilité est conservée) et généralisée (il y a stérilité) de 1'épididyme chez les boucs homozygotes $P P$ atteints de sténoses. En faisant correspondre à la clone des atteintes partielles notre clone des atteintes unilatérales, les atteintes bilatérales étant assimilées à la catégorie " atteintes généralisées " on a, à partir de nos données $\beta_{1}=0,43$ et $\beta_{2}=0,57$. Ces fréquences s'avèrent statistiquement différentes de celles que LAUVERGNE (I969) a évalué à partir des données de SchöNHERR (I954) $\left[\beta_{1}=0,638, \beta_{2}=0,362\right]$. Il est vrai que cet auteur n'adopte pas le même mode de classification que nous avons adopté; par ailleurs nous avons vu que nos données pouvaient être biaisées.

Ajoutons, pour terminer, que le diagnostic portant sur les diverses anomalies testiculaires est signalé aux éleveurs sans obligation d'en tenir compte et d'écarter de la reproduction les animaux atteints. Si les boucs hypoplasiques ou sténosiques 
bilatéraux s'éliminent d'eux-mêmes, par suite de leur stérilité, on n'a par contre aucune raison de penser que les boucs atteints de sténose unilatérale et, par conséquent, fertiles sont écartés, même partiellement, de la reproduction pour cette raison-là.

Reçu pour publication en octobre 1969.

\section{SUMMARY}

\section{MALE STERILITY IN POLLED SWISS GOAT BREEDS}

According to the methods of investigation of LöLIGER on the sterility of the he-goats the external genital organs of 966 young he-goats have been systematically examined during the show-markets of three swiss hornless breeds: Saanen, Toggenburg and Oberhasli-Brienz.

Two abnormalities were detected: - hypoplasia of the testicules and seminal congestion. The animals with hypoplasia had very small testicules, and did not produce semen or evidence any sex instinct. Seminal congestion was either one or two-sided, and located mainly in the epididymal heal. Some nodules the size of a nut mechanically prevented the semen from passing. Animals with hypoplasia or two-sided stenosis were sterile, one-sided stenosis becoming sometimes two-sided later on, and the fertile male changing into a sterile one. Abnormality frequencies were estimated as the following: 3.4 per cent hypoplasia, 7.6 per cent two-sided stenosis, 5.8 per cent one-sided stenosis. These figures are to some extent less than the real norm because stock-breeders are able to detect these abnormalities before sending the animals at the show.

We found abnormalities only in hornless breeds. As a check-sample, the examination of 175 horned Verzasca he-goats shows no example of abnormality.

Based on our date, the expressivities of partial and generalized bad effects among epididymal stenosic $P P$ males have been calculated as $\beta_{1}=0.43$ for the partial, and as $\beta_{2}=0.57$ for the generalized ones.

\section{RÉFÉRENCES BIBLIOGRAPHIQUES}

I,AUVERgne J. J., I969. Progrès des connaissances génétiques sur l'intersexualité associée à l'absence de cornes chez la chèvre d'origine alpine. Ann. Génét. Sél. anim., 1. 403-4I2.

LöLIGER H., I953. Beitrag zum Problem der Samenstauung. Fortpfl. Besam. Haustier, 3, 6-7, I2-I3. LöLIGeR H., r956a. Experimentelle Coli-Orchitis beim Ziegenbock. Arch. exp. Vet. Med,. 10, $582-588$. LöLIGER H., I956b. Unfruchtbarkeit bei Ziegenböcken und ihr Auftreten in der Schweiz. Kleinvichzuchter, 4, 576-580, 594-595.

LöLIGer H., r957a. Die Pathogenese der sog. Samenstauung beim Ziegenbock. Zentbl. Vet. Med., 4, 892-906.

I ÖLIGER H., r957b. Die Verbreitung von Unfruchtbarkeit unter den männlichen Tieren verschiedener Ziegenrassen in deutschen und ausländischen Zuchtgebieten. Zuchthyg., Fortpflstör. Besam. Haustiere, 1, 2OI-2I5.

ScHöNHERR S., r954. Die Unfruchtbarkeit der Ziegenböcke, ihre Verbreitung, frühzeitige Erkennung und Bekämpfung. Vet. med. Diss. Frei Univ. Berlin.

WEBER W., rg6r. Erfahrungen über die Sterilitätsuntersuchungen bei Ziegenböcken. Kleinvichzüchter, 9, 5 II-5I5.

WEBER W., r967. Unfruchtbarkeit bei Ziegenböcken. Kleinviehzuchter, 15, 899-9or. 